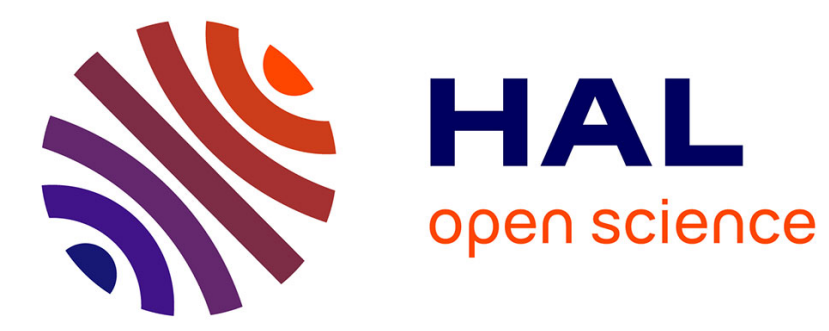

\title{
Sur un nouveau cercle à calculs
}

Pierre Weiss

\section{To cite this version:}

Pierre Weiss. Sur un nouveau cercle à calculs. J. Phys. Theor. Appl., 1901, 10 (1), pp.556-558. 10.1051/jphystap:0190100100055600 . jpa-00240554

\section{HAL Id: jpa-00240554 https://hal.science/jpa-00240554}

Submitted on 1 Jan 1901

HAL is a multi-disciplinary open access archive for the deposit and dissemination of scientific research documents, whether they are published or not. The documents may come from teaching and research institutions in France or abroad, or from public or private research centers.
L'archive ouverte pluridisciplinaire HAL, est destinée au dépôt et à la diffusion de documents scientifiques de niveau recherche, publiés ou non, émanant des établissements d'enseignement et de recherche français ou étrangers, des laboratoires publics ou privés. 


\title{
SUR UN NOUVEAU CERCLE A GALGULS;
}

\author{
Par M. Pierre WEISS.
}

Préoccupé de faire avec une dépense minima de temps et d'attention les calculs ordinaires de réduction des observations de Physique, jai été conduit à une disposition de cercle à calculs qui diffère d'ume manière assez marquée des cércles et des règles usuels, et dont un usage journalier m'a montré les avantages.

Linstrument comporte une seule graduation logarithmique, gravée sur métal, suivant une circonférence de 16 millimètres de diamètre. Cette graduation possède, comme celle de tous les autres cercles à calculs, la propriété de se juxtaposer à elle-même un nombre indéfini de fois. Elle est donc équivalente à une règle de longueur indéfinie, sur laquelle une échelle logarithmique, de 1 à 10, occuperait environ ö0 centimètres.

Sur celte graduation se meuvent deux aiguilles, que j'appellerai l'indicatrice et la multiplicatrice. L'indicatrice entraîne toujours dans son mouvement la multiplicatrice; celle-ci, au contraire, peut se mouvoir seule, sans déplacer l'indicatrice.

Toutes les propriétés de l'instrument sont évidentes, si l'on considère que, entre les nombres représentés par deux points distants, sur le cadran, diun angle constant, il y a un rapport constant. On multiplie donc les nombres indiqués par les deux aiguilles par un mème facteur, toutes les fois qu'on les fait tourner ensemble d'un même angle.

Pour faire un produit $a<b(f i g .1)$, on mettra lindicatrice sur lun des facteurs $a$, et la multiplicatrice, en la faisant mouvoir seule, sur la division 1. Puis on les fera tourner solidairement jusqu'à ce que la multiplicatrice soit en $b$. L'indicatrice se trouve alors en $a \ll b$.

On peut, sans lire ce premier produit, le multiplier immédiatement de la inème manière par un troisième facteur r, et ainsi de suite.

Pour diviser un nombre quelconque, par exemple le résultat des multiplications précédentes, que montre actuellement l'indicatrice, par un nombre $d$, on met la multiplicatrice, en la faisant mouroir seule, sur $d$, et l'on fait tourner les deux aiguilles d'un mouvement 
solidaire jusquà ce que la multiplicatrice soit en 1. Lð̈ndicatrice donne: $\frac{a<b>r}{d}$.

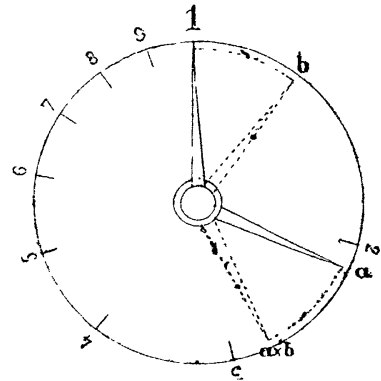

Firi. 1.

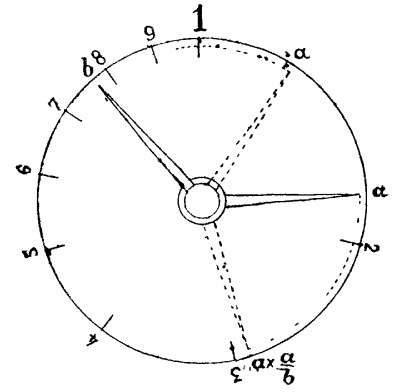

Fici. 2.

La position 1 intervient, on le voit, dans toutes ces opérations. On évite le pointé de cette position au moyen d'un butoir facultatif, qui entre en jeu ou est supprimé par un mouvement à ressort.

Dans le cas plus général ' $f g$. 2) de la multiplication d'un nombre * par un rapport $\frac{a}{b}$, la position 1 ne joue plus aucun rôle. On met lindicatrice en $\alpha$ et la multiplicatrice en $b$ en la faisant mouvoir seule. Puis on donne à l'ensemble des deux aiguilles un mouvement qui amène la multiplicatrice de $b$ en $a$. L'indicatrice donne alors $\alpha \times \frac{a}{b}$.

Quand on a des séries de nombres à multiplier par un même coefficient, on donne à l'angle des deux aiguilles la valeur correspondant à ce coefficient. Il suffit alors de mettre la multiplicatrice successivement en face de tous les membres, pour lire en face de l'indicatrice tous les produits.

En résumé, on peut, avec cet appareil, faire un nombre quelconque de multiplications et de divisions, sans que la précision et la rapidité des opérations soient diminuées par la leclure d'un résultat intermédiaire.

Les opérations ont un caractère de simplicité tel, qu'elles exigent beaucoup moins d'attention préalalle qu'avec la règle ordinaire. On nhésite, par exemple, jamais entre deux manieres de faire dont l'une risque de conduire en dehors des limites de la règle. Cette supériorité est encore plus marquée par rapport aux règles dans les- 
quelles l'échelle est repliée sur elle-mème afin de doubler la sensibilité pour une longueur de règle donnée.

La lecture elle-même est facilitée par la présence d'mine seule irhelle. On ne cherche pas lendroit où elle doit se faire sur une deuxième graduation voisine, mais on le trouve immédiatement au bout d'une aiguille. Quand même, dans la règle ordinaire, on se sert du curseur Mannheim, le voisinage de la deuxième graduation augmente un peu l'attention nécessaire.

Dans le même ordre d'idées, j'ai évité la complication de graduations donnant les racines carrées et les lignes trigonométriques, que l'on aurait dù mettre sur des cercles concentriques. Il m’a paru préférable de ne demander à un mème instrument que les opérations de beaucoup les plus fréquentes de la multiplication et de la division.

Ce cercle à calculs a été construit par M. I. Werlein. Les mouremenls, solidaires ou indépendants des aiguilles, s'obtiennent aisément, par les frottements gradués des douilles sur les axes, réglés une fois pour toutes. Chacune des aiguilles porte un bouton molleté, qui doit être assez grand pour que la manœuvre des aiguilles soit commode, et relativement léger pour éviter les effets de l’inertie.

On obtient très facilement, avec cet instrument, une précision minima de $\frac{1}{2000}$, mème dans les opérations compliquées. 